\title{
Upgrade project and plans for the ATLAS detector and trigger
}

\author{
F. Pastore* \\ Royal Holloway University of London (UK)
}

on behalf of the ATLAS Collaboration

\begin{abstract}
The LHC is expected to under go upgrades over the coming years in order to extend its scientific potential. Through two different phases (namely Phase-I and Phase-II), the average luminosity will be increased by a factor 5-10 above the design luminosity, $10^{34}$ $\mathrm{cm}^{-2} \mathrm{~s}^{-1}$. Consequently, the LHC experiments will need upgraded detectors and new infrastructure of the trigger and DAQ systems, to take into account the increase of radiation level and of particle rates foreseen at such high luminosity.

In this paper we describe the planned changes and the investigations for the ATLAS experiment, focusing on the requirements for the trigger system to handle the increase rate of collisions per beam crossing, while maintaining widely inclusive selections. In different steps, the trigger detectors will improve their selectivity by benefiting from increased granularity. To improve the flexibility of the system, the use of the tracking information in the lower levels of the trigger selection is also discussed. Lastly different scenarios are compared, based on the expected physics potential of ATLAS in this high luminosity regime.
\end{abstract}

Keywords: ATLAS, trigger, upgrade

\section{LHC Upgrade plans and physics goals}

The Large Hadron Collider (LHC) is designed as a dis- ${ }^{29}$ covery machine providing proton-proton collisions at a nominal $\sqrt{s}=14 \mathrm{TeV}$ and $\mathcal{L}=10^{34} \mathrm{~cm}^{-2} \mathrm{~s}^{-1}$. During 2011, the ${ }_{32}$ LHC provided collisions at reduced center-of-mass energy $\left(7_{33}^{32}\right.$ $\mathrm{GeV}$, upgraded to $8 \mathrm{GeV}$ during 2012), reaching about $3 \times 10^{33}$ $\mathrm{cm}^{-2} \mathrm{~s}^{-1}$ maximum luminosity. Its operational schedule foresees approximately a one-year long shutdown period every three years for major components upgrades. In the current plan, the ${ }_{37}$ first upgrade in 2013-2014 (18 months), referred as Phase-0, ${ }_{38}$ will allow to reach the design parameters and will be followed ${ }_{39}$ by Phase-I in 2018 which foresees main upgrades for the injector chain and the collimation system, in order to reach three ${ }_{41}$ times that luminosity. In 2022, an upgrade is planned to go well beyond the design limits with luminosity reaching $5 \times 10^{34}{ }_{43}^{42}$ $\mathrm{cm}^{-2} \mathrm{~s}^{-1}$ as leveled maximum, while maintaining the same col- ${ }_{44}^{43}$ lision energy. This project is referred as the High Luminosity LHC (HL-LHC) and corresponds to Phase-II upgrade.

The aim of such big increase of luminosity is mainly to pro- ${ }^{45}$ vide the experiments, like ATLAS, the required integrated luminosity (300 $\mathrm{fb}^{-1}$ after Phase-I and $3000 \mathrm{fb}^{-1}$ after Phase-II) ${ }^{46}$ for decreasing statistical errors on precision measurements and ${ }^{47}$ extending the physics potential with more convincing conclusions on signals at the limit of the sensitivity [1]. HL-LHC upgrade for example will give a conclusive understanding of the ${ }_{51}^{50}$ Standard Model Symmetry Breaking mechanism. If Higgs is ${ }^{5}$ discovered, $300 \mathrm{fb}^{-1}$ will allow the observation of many of its ${ }_{53}^{52}$

${ }^{*}$ Corresponding author

Email address: francesca.pastore@cern.ch (F. Pastore) decay modes, while 10 times that luminosity will increase the sensitivity on the measurement of its parameters (mass, width, cross-sections and main couplings). Moreover the scenario can be enlarged by including the quest for physics beyond the Standard Model that can show up in the high mass regime.

HL-LHC upgrade could push mass reach by typically $20 \%$ with no major detector changes. However, with upgraded detectors one could fully benefit from luminosity increase. This is the motivation for the design requirements of both preserving good performance and ensuring flexible and robust physics selection at this high luminosity regimes. Even if the picture is not clear today, many of these scenarios involve objects at (near) the electroweak scale, like leptons with $20 \mathrm{GeV} / \mathrm{c}$ transverse momentum $\left(\mathrm{p}_{T}\right), b$-meson and $\tau$-lepton tagging as well as missing transverse energy. So the physics selections must reflect these scales in order to avoid reduced acceptance and poor efficiency.

\section{The ATLAS trigger at High Luminosity}

The ATLAS experiment [2] has been designed to operate at the nominal LHC parameters, with small additional margins. In the HL-LHC high radiation environment, some of the detector constituents (calorimeter electronics, silicon trackers) are subject to aging, mainly in the forward regions, while others, like the muon chambers, will suffer from increasing occupancy and will degrade their performance. Furthermore the trigger system will have to make tighter selection requirements in a more difficult environment. Phase- 0 and Phase-I ATLAS upgrades are already documented with a Letter of Intent [3], while the main steps for Phase-II are currently under study. 
The ATLAS Trigger and Data Acquisition (TDAQ) system is organized in a three-level selection scheme, including the hardware-based first-level (L1) trigger and the higher levels (L2 and Event Filter EF) implemented as software systems distributed on commodity nodes. Despite its extremely good performance (95\% DAQ efficiency during 2011 [4]), some limits are expected at higher luminosity. The guidance for upgrade decisions is set by the gained experience during the first years of data taking. Extrapolations from data and simulations (with adequate safety factors) help in assessing the feasibility of increasing rejection power. This can be achieved with both improvements on the algorithms and possible modest changes in the current TDAQ system, which additionally demand that any component installed in Phase-I must be fully operational also through Phase-II.

In order to maintain inclusive and unbiased selections at the EW scale, we use as a guideline for understanding the total L1 bandwidth requirement at HL-LHC the current sharing of the bandwidth, where $40 \%$ is reserved for high $\mathrm{p}_{T}$ electrons and muons $\left(20 \mathrm{kHz}\right.$ each over the full $100 \mathrm{kHz}$ budget with the cur- ${ }_{-114}$ rent system). In addition to the trivial increase of trigger rates ${ }_{115}$ due to the corresponding increase of luminosity, a major im-116 pact on the trigger system is expected from the higher number ${ }_{117}$ of interactions per collision (pile-up). Extrapolations of trig-118 ger rates are periodically done now during data-taking, when ${ }_{119}$ ATLAS has to face planned increases of luminosity. They are ${ }_{120}$ needed to assess the correct sharing of the bandwidth, using ${ }_{121}$ various handles: increasing the thresholds and tightening the ${ }_{122}$ isolation and the identification criteria on single leptons; opti-123 mizing the selections of global-event signatures, like jets and ${ }_{124}$ missing-energy, whose rates grow exponentially due to pile-up ${ }_{125}$ and are more complicated to predict; including more exclusive selections to save low momentum spectra. At HL-LHC we expect about 200 minimum bias collisions each $25 \mathrm{~ns}$ bunch $^{126}$ crossing, with order of ten thousand particles, mostly with low ${ }_{127}$ momentum. The higher occupancies in the detectors strongly ${ }_{128}$ reduces the rejection power of the algorithms because of worse ${ }_{129}$ energy resolution in the calorimeters and less effective isolation ${ }_{130}$ and pattern recognition. One can also expect larger event size, ${ }_{131}$ which consequently reduces the maximum rate at $\mathrm{L} 1$ for fixed ${ }_{132}$ bandwidth. In parallel, the rise in fake rates increases the rates ${ }_{133}$ expected from double-object selections.

A simple increase of thresholds can reduce the signal dras-135 tically: for example, Fig. 1 shows how increasing the muon ${ }_{136}$ threshold from 20 to $40 \mathrm{GeV} / \mathrm{c}$ halves the acceptance for leptons ${ }_{137}$ produced in electroweak boson decays. Hence more sophisti-138 cated trigger criteria are needed, based on the full understand- ${ }_{139}$ ing of the background sources, which are mainly coming from ${ }_{140}$ jets mimicking electrons and high hit rates from ambient radi-141 ation in the forward muon chambers. The trend is to use algo-142 rithms and resolutions at L1 similar to those currently achieved ${ }_{143}$ in the higher trigger levels, while those latter would increase ${ }_{144}$ their complexity. At any level, it may require both longer laten-145 cies, since reconstruction complexity and timing naively scale ${ }_{146}$ with the number of tracks, and higher data throughputs, due to ${ }_{147}$ the larger request of data. We get help from technology to pro-148 vide the systems increased bandwidth, longer timing and flexi-149

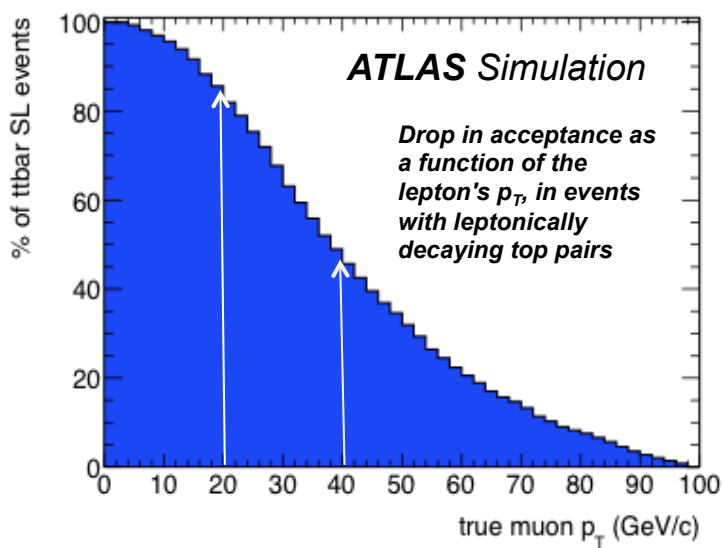

Figure 1: Fraction of $\mathrm{t} \overline{\mathrm{t}}$ events selected by one muon in the final state from the $\mathrm{W}$ decay, with momentum greater than the given threshold. The arrows show the reduced acceptance on these events when the momentum threshold is raised from 20 to $40 \mathrm{GeV} / \mathrm{c}$.

bility. They can profit from the high level of parallelism (parallel processing, multi-cores and GPUs), the dramatic increase in computing power and $\mathrm{I} / \mathrm{O}$, the development of chips with increasing densities, and reduced size, and the development of radiation resistant electronics. Modern fast FPGAs, with huge processing capabilities, and fast connections, like optical links up to 10 Gbit speed, are example of the technology improvements that can handle the increased demands. The current upgrade plans are mainly devoted to increase the robustness of the L1 systems, which need longer hardware development, while the high level trigger systems, being software based, are surely more flexible.

\section{Main plans for L1 Upgrades during Phase-0 and Phase-I}

During Phase-0 upgrade (2013), the ATLAS L1 trigger will be equipped with a new Central Trigger Processor (CTP) in order to allow the use of topological criteria and more exclusive selections. This board will mainly have increased number of inputs, and combination of them, limited in this first phase to those coming from the calorimeters. The muon signals will probably be merged later on.

Phase-I upgrades must cope with $\mathcal{L}=3 \times 10^{34} \mathrm{~cm}^{-2} \mathrm{~s}^{-1}$. No major architectural changes to the detector read-out and DAQ are planned up to this phase, so the system stays with current design limits: L1 decision latency less than $2.5 \mu \mathrm{s}$ (+ spare $\sim 0.5 \mu \mathrm{s}$ ) and average accept rate less than $100 \mathrm{kHz}$. Without any improvement, the predictions at that luminosity estimate $120 \mathrm{kHz}$ of isolated electrons above $23 \mathrm{GeV}$ and $60 \mathrm{kHz}$ of isolated muons above $20 \mathrm{GeV} / \mathrm{c}$. Two main upgrades, increasing the flexibility of the system, are thus foreseen.

In the muon system, the $\mathrm{L} 1$ rate will be reduced drastically in the forward region by the addition, in the trigger coincidence, of one layer in the innermost region of the spectrometer (New Small Wheel, NSW). Here, only $2 \%$ of the L1 triggers at 20 $\mathrm{GeV}$ threshold contains a high quality muon. The request of coincidence hits in the innermost region will reject the big contribution of low momentum particles produced along the beam 


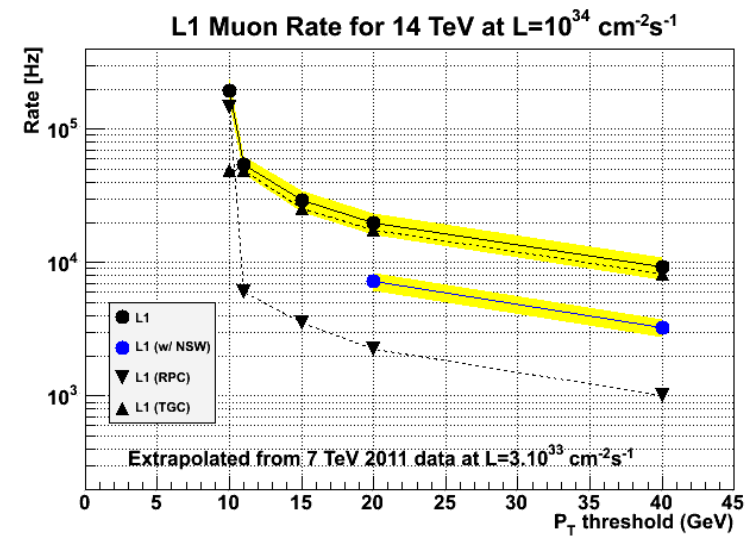

Figure 2: Expected L1 muon rates at $\mathcal{L}=10^{34} \mathrm{~cm}^{-2} \mathrm{~s}^{-1}$ as a function of the momentum threshold, with different detector layouts (with and without the NSW). The error bands show the extrapolations from 7 to $14 \mathrm{TeV}$ uncertainty. In the plot are also shown the contributions to the rate from the Endcaps $(\eta>1$, Thin Gap Chambers) and the Barrel (Resistive Plate Chambers) regions.

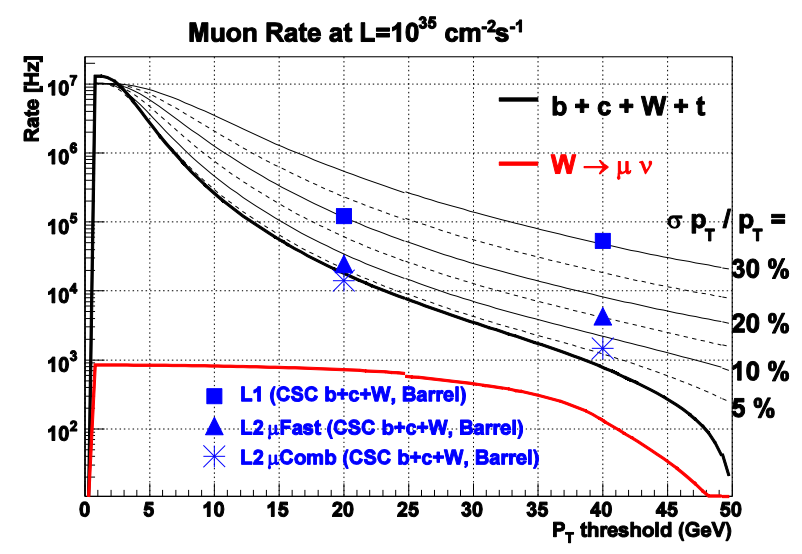

Figure 3: Expected L1 muon rates at $\mathcal{L}=10^{35} \mathrm{~cm}^{-2} \mathrm{~s}^{-1}$ as a function of the mo-179 mentum threshold, calculated by convoluting the physics cross-sections with ${ }_{180}$ different $\mathrm{p}_{T}$ resolutions, and compared with full simulation extrapolated from lower luminosities. Inclusive L1 rate is over $50 \mathrm{kHz}$ at $40 \mathrm{GeV} / \mathrm{c}$ threshold with ${ }^{181}$ current $30 \%$ resolution.

line and not coming from the interaction region. Studies have ${ }_{185}$ been done, as shown in Fig. 2, to evaluate the expected rejection ${ }_{186}$ factor. Depending on the applied algorithms, the $20 \mathrm{GeV}$ muon $_{187}$ rate is expected to be reduced between factor 3 to 10 . More- ${ }_{188}$ over this layer will be equipped with a new detector, based ${ }_{189}$ Micromega and small-TGC technology, replacing the current 190 MDT/CSC system which will suffer from too high level of ra- ${ }_{191}$ diation at this luminosity. An increased angular (and $\mathrm{p}_{T}$ ) reso- ${ }_{192}$ lution on these detectors will give additional handles for rate ${ }_{193}$ control, as visible in Fig. 3.

194

The L1 calorimeter trigger will undergo a partial replacement 195 of the trigger and readout electronics, which will allow the trig- ${ }_{-196}$ ger algorithms to access information with increased granularity 197 (the trigger towers will be reduced by a factor four in the $\eta$ di-198 rection). In addition to isolation criteria, other algorithms could ${ }_{199}$ be applied with good rejection, based on electromagnetic (EM) 200 shower shapes and depth information from both hadronic and 201

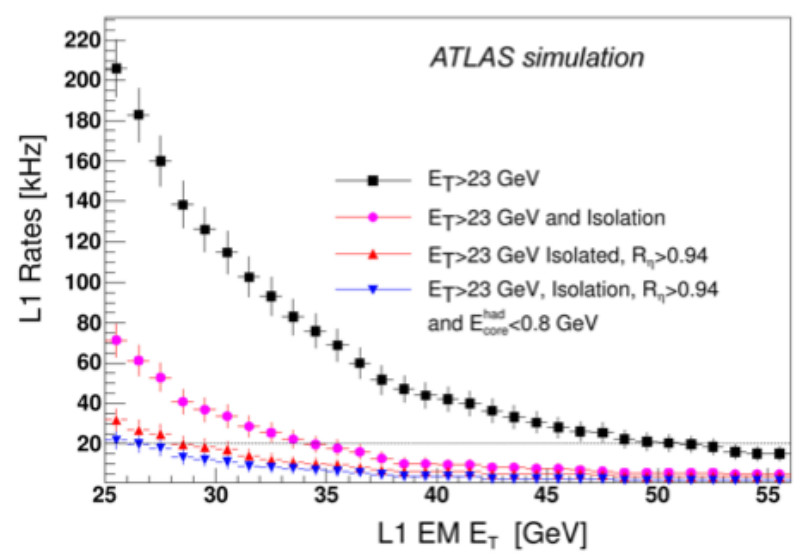

Figure 4: Expected L1 EM rates at $\mathcal{L}=10^{34} \mathrm{~cm}^{-2} \mathrm{~s}^{-1}$ as a function of the energy threshold for different algorithms and with the current trigger system. Note in particular the effect of reducing rate of isolated EM when applying cuts on the shower shapes $\left(R_{\eta}\right)$ and the hadronic core energy $\left(E_{\text {core }}^{\text {had }}\right)$.

EM parts. In particular these new algorithms will help in enhancing the purity of the electron selection, by rejecting low $\mathrm{p}_{T}$ jets by factor 3 to 5 as shown in Fig. 4, with additional expected improvements in the $\tau$ and missing $E_{T}$ measurements. This plot also indicates the thresholds required for a $20 \mathrm{kHz}$ allocated bandwidth to EM triggers: with these new conditions the threshold can be maintained below $25 \mathrm{GeV}$.

\section{Evolution of L1 trigger Upgrades during Phase-II}

The plans for Phase-II are less fixed than those for Phase-I. With these upgrades, ATLAS must be prepared for maximum $\mathcal{L}=7 \times 10^{34} \mathrm{~cm}^{-2} \mathrm{~s}^{-1}$, having the challenge of controlling the increased trigger rates at acceptable thresholds. In the current system, to limit the non-isolated EM rates below $20 \mathrm{kHz}$, as shown in Fig. 5, the energy threshold should be raised over 50 $\mathrm{GeV}$, with radical effects on the physics potentials. In particular, at this luminosity, and including the upgrades already planned for Phase-I described above, the lepton rates will exceed the allowed maximum: inclusive EM rates are expected to be over $100 \mathrm{kHz}(500 \mathrm{kHz}$ reduced by factor 5 in Phase-I) and muon rates over $50 \mathrm{kHz}(\sim 140 \mathrm{kHz}$ reduce by factor 3 in Phase-I).

The calorimeter and the muon systems need then to be changed accordingly, in order to refine the L1 algorithms. One approach is to further increase the granularity, and thus the resolution, of each system, as it's currently done at L2 trigger: by using the information from the full calorimeter readout and, in the muon spectrometer, from the precision chambers (MDTs). Some studies are on-going to understand the limits of such approaches in the current systems (the entire calorimeter electronics will be replaced anyway) and evaluate the size of the required changes. Additionally the use of tracking information at L1 is also considered, which would give the system higher degree of flexibility against unexpected scenarios. Combining the calorimeter/muon information with tracks identified in the inner silicon detectors (which will be upgraded anyway) will allow to improve the corresponding selections. Current studies 
Thresholds for non isolated EM objects vs. Inst. Luminosity @ 20kHz Level-1 Trigger rate

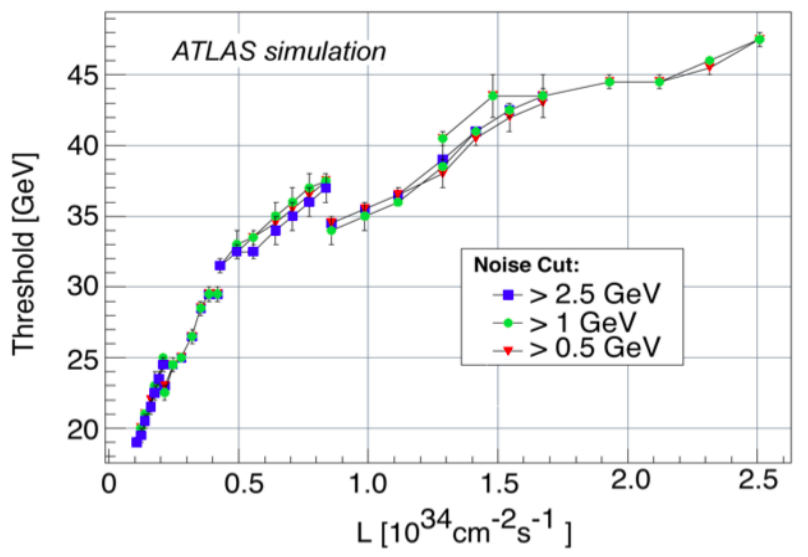

Figure 5: EM thresholds required to limit the L1 EM trigger rate to $20 \mathrm{kHz}^{234}$ as a function of instantaneous luminosity. The different curves correspond to ${ }^{235}$ different levels of noise suppression thresholds.

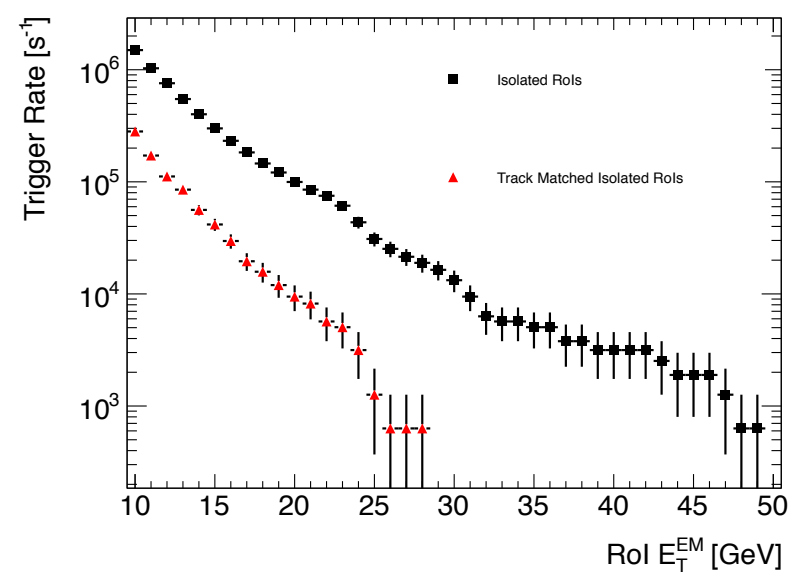

Figure 6: Expected isolated EM rate reduction as a function of the energy ${ }^{253}$ threshold, when applying EM-track match at $14 \mathrm{TeV}$ and $\mathcal{L}=3 \times 10^{34} \mathrm{~cm}^{-2} \mathrm{~s}^{-1}$. 254 show that the EM rate can be reduced by factor 10 (as shown in Fig. 6) and the muon rate by factor 5, when applying this additional request. Moreover having the trackers information ${ }^{257}$ available at L1 would provide powerful tools like track isolation (more reliable at high pile-up conditions), track multiplicity for ${ }^{258}$ $\tau$ identification and impact-parameter for $b$-tagging.

In order to readout such increased amount of information, ${ }^{26}$ both approaches, which are not exclusive, may require high ${ }^{261}$ data bandwidths and extended L1 latency, which are over the ${ }^{262}$ current design limits. The increase of the maximum L1 rate ${ }^{263}$ above $200 \mathrm{kHz}$ could have severe implications, like rebuilding ${ }^{264}$ the $\mathrm{L} 1$ processors and some front-end electronics. The increase of the L1 decision latency up to $20 \mu$ s is expected to be feasible 265 for all detectors and to provide enough time to include tracking information. Given the long timeline of this luminosity upgrade ${ }_{267}^{266}$ beyond 2022, major changes to the TDAQ architecture can be ${ }_{268}$ foreseen. The new design must additionally accomplish other 269 requirements, like weighing the benchmark physics channels, ${ }^{270}$ the changes in the detectors (mainly for the trackers) and the eventual demands from the data-flow system (change of size and segmentation of the events). An alternative scenario is also proposed, which foresees a two-stage hardware trigger: a L0, including the current L1 systems with a higher accept rate (up to $500 \mathrm{kHz}$ ), followed by a further hardware trigger decision (L1) with a significant longer latency (10-30 $\mu$ s). This approach would allow to minimize the buffer size needed for long latency decisions and accommodate the inclusion of the tracking information without affecting the tracker design. The decisions on such changes in the architecture are currently being considered within ATLAS and options will be defined in the Letter of Intent expected to be completed in 2012 .

\section{High level triggers evolution}

A gradual upgrade program is foreseen for the high level triggers. Mostly based on commodity components (excepts the Readout System ROS), the changes here will follow the technology development very closely. There will be increasing demands on resources (network and CPUs) and on the complexity of the system (more TDAQ applications), which will involve issues for configurations, control and monitoring. For next year upgrade (Phase-0) the main concern is to eliminate bottlenecks, mainly on network traffic. Studies are on-going to: increase the density of the readout links for higher speed ROS system; assess the feasibility of using GPUs for the L2 tracking; optimize the L2/EF resources and networks by allowing both stages to share the same hardware node. Moreover, over longer terms, new hardware preprocessors will be integrated into the TDAQ. During Phase-I, a Fast Tracker Trigger (FTK) [5], completely hardware based (track finder with AM technology) will provide tracking parameters (close to offline resolution) at full L1 rate within $\mathrm{O}(100 \mu \mathrm{s}) \mathrm{L} 2$ latency. It will allow to enhance the capability for $b / \tau$ tagging and lepton isolation and optimize the L2 selection with more complex software selections. Phase-II evolutions for the high level triggers are not considered yet, but may involve radical changes of the architecture, like changes of the number of the physical trigger levels (in both directions).

\section{Conclusions}

The ATLAS trigger upgrade program has many interesting technical challenges in the high luminosity scenarios promised by LHC during the next 10 years and above. While Phase-I changes are already planned, for HL-LHC we are investigating potential benefits of alternative technologies and strategies to maximize our ability to access the interesting collisions provided by the LHC.

\section{References}

[1] F. Gianotti et al., CERN-TH/2002-078, hep-ph/0204087, April 1, 2002.

[2] ATLAS Collaboration, JINST 3 S08003 (2008).

[3] ATLAS Collaboration, CERN-LHCC-2011-012.

[4] ATLAS Collaboration, Eur. Phys. J. C 72 (2012) 1849.

[5] A. Annovi et al., IEEE Trans. Nucl. Sci. 51 (2004) 391. 